\title{
A method to estimate the environmental impacts from genetic change in pig production systems
}

\author{
Mathias Ottosen $^{1}$ (D) $\cdot$ Stephen G. Mackenzie ${ }^{1} \cdot$ Michael Wallace $^{1} \cdot$ Ilias Kyriazakis ${ }^{1}$
}

Received: 12 April 2019 / Accepted: 5 September 2019 / Published online: 14 November 2019

(C) The Author(s) 2019

\begin{abstract}
Purpose The environmental impacts (EIs) of the global pig production sector are expected to increase with increasing global pork demand. Although the pig breeding industry has made significant progress over the last decades in reducing its EI, previous work has been unable to differentiate between the improvements made through management improvements from those caused by genetic change. Our study investigates the effect of altering genetic components of individual traits on the EI of pig systems. Methods An LCA model, with a functional unit of $1 \mathrm{~kg}$ live weight pig, was built simulating an intensive pig production system; inputs of feed and outputs of manure were adjusted according to genetic performance traits. Feed intake was simulated with an animal energy requirement model. A correlation matrix of the genetic variance and correlations of traits was pooled from data on commercial pig populations in the literature. Three sensitivity analyses were applied: one-at-a-time sensitivity analysis (OAT) used the genetic standard deviations, clusters-of-traits sensitivity analysis (COT) used the genetic standard deviations and clustering based on correlations, and the sensitivity index (SI) applied the full correlation matrix. Five EI categories were considered: global warming potential, terrestrial acidification potential, freshwater eutrophication potential, land use, and fossil resource scarcity.

Results and discussion The different EI categories showed similar behaviour for each trait in the sensitivity analyses. OAT showed up to $18 \%$ change in EI relative to baseline for energy maintenance and around 3\% change in EI relative to baseline for most other traits. COT grouped traits into a grower/finisher cluster (up to $17 \%$ change relative to baseline), a reproductive cluster (up to $7 \%$ change relative to baseline), and a sow robustness cluster (up to $2 \%$ change relative to baseline), all clusters including negative correlations between traits. By including genetic correlations, the SI went from being influenced by maintenance, and finisher and gilt growth rate into solely being dominated by maintenancen and protein-to-lipid ratio responsible for above 0.8 and 0.35 of the variance in EI respectively.

Conclusions We developed a novel methodology for evaluating EIs of changes in correlated genetic traits in pigs. We found it was essential to include correlations in the sensitivity analysis, since the local and global sensitivity analyses were not affected to the same extend by the same traits. Further, we found that finisher growth rate, body protein-to-lipid ratio, and energy maintenance could be important in reducing EI, but mortalities and sow robustness had little effect.
\end{abstract}

Keywords Breeding $\cdot$ Environmental impact $\cdot$ Genetic traits $\cdot$ Life cycle assessment $\cdot$ Pigs $\cdot$ Sensitivity analysis

\section{Introduction}

Responsible editor: Thomas Jan Nemecek

Electronic supplementary material The online version of this article (https://doi.org/10.1007/s11367-019-01686-8) contains supplementary material, which is available to authorized users.

Mathias Ottosen

m.ottosen2@newcastle.ac.uk

1 Agriculture, School of Natural and Environmental Sciences, Newcastle University, Newcastle upon Tyne, UK
Pork is one of the most consumed meat products worldwide (FAOSTAT 2018) and the demand for pig meat is expected to increase with the growth in global population and economy (FAO 2017). At the same time, there is growing consensus that the current environmental impacts of livestock systems, including pig systems, are unsustainable (Springmann et al. 2018). Considerable evidence has also been presented that through changes to production systems, these impacts can be reduced (Herrero et al. 2016; Poore and Nemecek 2018). Most 
environmental impacts from pig production come from feed production, but manure management also makes major contributions (McAuliffe et al. 2016; Monteiro et al. 2016). Therefore, changes in pig performance traits that affect inputs or outputs of a pig production system can have a substantial influence on the environmental impact of their production systems.

Sixty percent of the global pork production is produced in intensive indoor farming systems (MacLeod et al. 2013). In most commercial settings, farms continuously receive improved genetics from pig breeding companies, either as semen or new breeding stock (Knox 2016). Current pig performance has resulted from large changes in the main traits during the last decades, due to intensive selective pressure (Tribout et al. 2010). Previous life cycle assessment (LCA) studies investigating the environmental impacts of pig and other meat production systems over time have suggested big reductions in impact per kilogram of output produced (Capper et al. 2009; Cederberg et al. 2009; Verge et al. 2009; Capper 2011; Pelletier et al. 2014; Pelletier 2018). However, in all these studies, efficiency improvements across the production system are included in the emission reductions, and it is therefore not possible to differentiate between improvements from changes in genetic selection, improved management and feeding, or upstream efficiency improvements in feed and fertilizer production, transport, and energy generation. Here, we present a method for estimating the environmental effects of breeding by accounting for changes in genetic traits in pigs.

In animal breeding, only the phenotype of an individual animal can be directly measured. However, the effect of an individual trait can be estimated on a population level, since different traits will lead to different performance of different animals. Under the assumption of a constant environment and no environment-gene covariance, the effect of the genetic difference in individual traits can be estimated mathematically in a given population, and the effect of the specific genetic combination can be estimated at animal level (Oldenbroek and van der Waaij 2014). In this paper, the term genetic traits (GTs) will be used to describe the phenotypical effect of the genetic difference in individual traits in a given animal.

It is often problematic to include correlations between input variables in LCA modelling (Wei et al. 2015) and it is therefore common for studies to ignore correlations, notwithstanding their potentially significant influence on model results. However, the risk of under or overestimating the variance and sensitivity of the system, by ignoring correlations in LCA modelling, can be substantial (Groen and Heijungs 2017). The model presented in this paper used GTs in pigs as independent input variables. Since it is well known that the genetic component of some traits are strongly correlated (e.g. feed efficiency which is both dependent on the diet and about a dozen metabolic and growth traits (Reckmann and Krieter 2015)), it was unreasonable to assume that GTs in pigs are uncorrelated. In this paper, we developed an LCA model that implements correlations between GTs when quantifying the environmental impacts of pig systems.

The aim of the study was to develop a methodology that could (1) evaluate the environmental impacts of each GT in pigs, (2) fully implement the available data on correlations between the genetic components of traits in the present pig populations, and (3) investigate the effect of including correlations on the outputs of an animal LCA model. To fulfil these objectives, we constructed a cradle to farm-gate LCA model of a pig farming system, which takes the most common unambiguous GTs for pigs as independent variables. A correlation matrix was compiled from literature, and the sensitivity of the LCA to each GT was tested with local and global sensitivity analyses. We hypothesise that not all GTs included in common pig breeding objectives are able to reduce the environmental impacts of pig systems to the same extend. Although the model was developed for and applied to pigs, it is expected that the methodology could be applied to other livestock systems to explore similar questions.

\section{Materials and method}

\subsection{Goal and scope}

The goal of this study was to investigate how GTs in pigs can be included in a livestock LCA framework. Further, we wanted to explore which GTs influenced the environmental impacts of the system and how their interactions could affect future breeding goals in pigs. The study boundaries were cradle to farm gate and the functional unit was $1 \mathrm{~kg}$ of live animal at the farm gate (FAO 2016). The functional unit was chosen to avoid uncertain assumptions on the killing out percentage and the meat quality which are certainly affected by traits but are difficult to estimate from animal modelling. The model used attributional LCA (BSI 2011) and distributed impacts among coproducts by economic allocation (Mackenzie et al. 2017).

All EI categories from the ReCiPe 2016 midpoint method (Huijbregts et al. 2016) were considered in this study. To align with FAO (2018), all impact categories with sufficient available data were calculated and the most important impacts are reported and discussed in this paper: global warming potential (GWP), terrestrial acidification potential (TAP), freshwater eutrophication potential (FEP), land use (LU), and fossil resource scarcity (FRS). ${ }^{1}$ Available impact categories that are not commonly reported in animal LCA studies are reported in the Electronic Supplementary Material - ESM, section 8.13: Stratospheric Ozone Depletion, Ionizing Radiation and

\footnotetext{
${ }^{1}$ This impact category incorporates fossil energy use in its characterization as referenced in Table 2 of the LEAP guidelines for pig systems (FAO 2018)
} 
Mineral Resource Scarcity. Due to data unavailability, ozone formation, fine particulate matter, toxicity, and water consumption impacts were not calculated.

\subsection{Choice of system}

An intensive indoor pig production system was chosen since most of the pigs produced in Europe are raised as such (Driver 2017). The Danish pig production industry has published abundant information which, together with the influential Danish contribution to the European pig semen and pork market, gave an opportunity for detailed modelling of a highly defined, representative system that is recognized as an international benchmark. To evaluate a more holistic perspective of the influence of GTs on the full life cycle of the pig, an integrated pig production system, which contains both pig breeding and a production unit, was chosen. In this paper, the Baseline System will be used for the unmodified system where all variables have their mean value, and the Baseline System is therefore deterministic.

\subsection{The system}

The model covered 3 stages in the life of the animals: (1) gilt: female pigs from weaning until the point of mating, where the gilts were fed together with the production pigs until day 56 and thereafter fed a specialist gilt diet; (2) sow: female pigs from first insemination until culling including piglets until weaning; this stage includes gestation, lactation, wean to oestrus, and failed pregnancies; (3) production pig: growing and finishing of pigs from weaning to slaughter weight at farm-gate (see Fig. 1). The terms gilt, sow, and production pig will be used in the following text to refer to these three stages. Male reproducing pigs were not included in the model since preliminary calculations estimated that thousands of offspring may result from a single boar ( $>3000$ and $>95,000$ for natural and artificial insemination, respectively) and their influence on the slaughtered pigs could, therefore, be expected to be minimal. Time spent in each stage, diet composition, and feed amount for the Baseline System can be found in the ESM, section 8.1-8.3.

The LCA model was constructed to simulate the integrated Danish intensive indoor pig system based on the method described by Mackenzie et al. (2015) (see Fig. 1). The structure of the system was based on recommendations for pig management published on the home page of the national Danish Pig Board (SEGES 2018). A sow compartment, containing the first two life stages, following the sow from birth to 8th parity, was constructed, with feed, manure, and litter sizes adjusted according to age and surviving proportion of sows (calculated from phase specific mortality rates) (see section 8.1-8.3). A production pig compartment, equivalent to the third life stage, followed the pig from birth to slaughter at $110 \mathrm{~kg}$, with feed intake and output of live pig adjusted for mortality rates. A single feed was chosen from weaning to slaughter for the production pigs since phase feeding is not commonly applied in Denmark (Sloth 2000) and trials on phase feeding have previously shown reduced leanness in Danish trials (SEGES 2013a). Diet was composed of typical commercial feed ingredients, with 6 different feeds (creep feed, production pig feed, gilt feed, early gestation feed, late gestation feed, lactation feed) offered to the sow and 2 different feeds (creep feed, production pig feed) offered to the growing pigs, according to published information and the recommendations for the Danish pig industry. The composition of all feeds can be seen in the ESM, section 8.1. Emissions from feed ingredients and power consumption on farm were taken from inventories for Denmark or similar countries from the SimaPro 8.5.2.0 database (Pre Consultants 2017). Manure chemical composition was calculated based on the mass balance principle, and emissions from the pig unit and manure management were calculated based on (IPCC 2006) for GWP and (Guinee 2002) for other chemicals.

\subsection{Pig traits considered in the LCA model and their origin}

To account for the performance of different pig genotypes, a number of trait variances were included for in the modelling process to supplement the baseline model (see Table 1). In the Baseline System, all traits had their average value. When calculating the outcomes of the model and the sensitivity analyses, a selected number of standard deviations (SD) were added to the average value (see section 2.9 for details). Included traits were chosen to account for different parts of the energy dynamics and consumption of the pig, and to account for changes in inputs or outputs of the model. Traits with very small genetic variance relative to the Baseline System (such as gestation length-variance around 1 day) and traits which no genetic variance reported in literature (such as digestive efficiency) were not included in the analyses. The genetic variance and covariance were taken from different sources in the literature and the pooling method can be seen in detail in 2.9 and in the ESM, section 8.12. Average daily gain from birth to $30 \mathrm{~kg}$ (ADG30) and average daily gain from 30 to $100 \mathrm{~kg}$ (ADG100) were treated as two different traits, since the breeding literature often reports them separately, even though they are not independent. ADG100 was taken from literature which reported growth rate from approximately $30 \mathrm{~kg}$ until normal slaughter weight at around $100 \mathrm{~kg}$. Due to the many different end weights for the late growth rate and for simplicity reasons, a single number, 100 , was chosen to specify the traits which covered rearing to finishing weight. Sow age at sexual maturity (AgeMature) and sow body weight at sexual maturity (BWMature) were both taken from literature reporting days to or body weight at sexual maturity respectively at first 




Fig. 1 Schematic model of the pig production system considered. The reproductive system, consisting of the gilt and the sow, produces piglets for each parity. These piglets are used to grow replacement gilts and

insemination. Body protein-to-lipid ratio (ProtLip) was approximated based on the variance in reported lean meat, and calculated from protein concentration in the empty body and organs reported by Szabó et al. (2001) (see ESM, section 8.5). Average number of piglets per litter (LitterSize) was treated as a single trait, even though there are clear differences in sow performance during different parities, but data on correlations slaughter pugs. Manure is managed on farm and spread on fields where it replaces artificial fertilizers

for a trait for each parity was not available. The model incorporated post weaning mortality (PostWMort) with a different variance for rearing and finishing pigs, but since correlations were only available for a single post weaning mortality trait, they always had the same number of SD added to the average trait value. Daily metabolic energy maintenance (Maintenance) was based on reported residual feed intake
Table 1 Abbreviations and description of the pig performance traits considered; the standard deviation (SD) and the relevant units are also given. PostWMort was considered a single trait with two different variances. All input values and trait calculations in the model can be found in the supplementary materials section $8.1-8.8$

\begin{tabular}{|c|c|c|c|c|}
\hline Abbreviation & Trait & Mean & Source & $\begin{array}{l}\text { Standard } \\
\text { deviation }\end{array}$ \\
\hline ADG100 & Average daily gain from $30 \mathrm{~kg}$ to $100 \mathrm{~kg}$ & $0.879 \mathrm{~kg} /$ day & (SEGES 2012) & $0.052 \mathrm{~kg} /$ day \\
\hline ADG30 & Average daily gain from birth to $30 \mathrm{~kg}$ & $0.461 \mathrm{~kg} /$ day & (SEGES 2012) & $0.015 \mathrm{~kg} /$ day \\
\hline AgeMature & Sow age at sexual maturity & 215 days & (Miller et al. 2011) & 25.3 days \\
\hline AMPar & Average maximum sow parity & 4.32 parities & (SEGES, n.d.) & 0.74 parity \\
\hline BWLossLactation & Sow body weight loss during lactation & $\begin{array}{l}\text { Reproductive cycle gains and } \\
\text { losses }=20 \mathrm{~kg}\end{array}$ & (SEGES 2013b) & $6.160 \mathrm{~kg}$ \\
\hline BWMature & Sow bodyweight at sexual maturity & $148 \mathrm{~kg}$ & (Miller et al. 2011) & $17.717 \mathrm{~kg}$ \\
\hline LactationFeed & Sow feed intake during lactation stage & $170-208 \mathrm{~kg}$ & (Sørensen 2005) & $7.789 \mathrm{~kg}$ \\
\hline LitterSize & Average number of live born piglets per litter & $14.2-16.2$ depending on parity & (SEGES, n.d.) & 0.81 piglet/litter \\
\hline Maintenance & $\begin{array}{l}\text { Daily metabolic energy maintenance, "a" in the } \\
\text { equation } E=a * B W^{b}\end{array}$ & $0.44 \mathrm{MJ} / \mathrm{kg}^{0.75}$ & (Dourmad et al. 2008) & $0.073 \mathrm{MJ} / \mathrm{kg}^{0.75}$ \\
\hline $\begin{array}{l}\text { PostWMort } \\
\text { Rearing: }\end{array}$ & $\begin{array}{l}\text { Mortality of pigs alive at weaning until slaughter } \\
\text { weight }\end{array}$ & $3.1 \%$ & $\begin{array}{l}\text { (SEGES, personal } \\
\text { communication) }\end{array}$ & $3.2 \%$ points \\
\hline Finisher: & & $3.3 \%$ & & $3.4 \%$ points \\
\hline PreWMort & Mortality for live born piglets until weaning & $12 \%$ & (SEGES, n.d.) & $7.5 \%$ points \\
\hline ProtLip & Protein to lipid ratio in the body of the animal & 0.50 at maturity & Result of diet & 0.0087 \\
\hline
\end{tabular}


from rearing to slaughter weight and the equivalent energy was scaled to metabolic body weight to fit the rest of the life cycle of the animal (see ESM, section 8.5). Sow average maximum parity (AMPar) was chosen to represent the robustness of the sow, since it has been reported at a much higher frequency and was easier to model than lifetime or fitness of the sow.

\subsection{Energy balance model}

The pig model specified inputs and outputs as functions of GT, and feed intake was based on energy demand from growth and maintenance. Feed intake was calculated from the required energy for each day based on (Wellock et al. 2003; Dourmad et al. 2008; Tallentire et al. 2016) with daily energy intake calculated as

$E_{f} \times R_{d}=E_{m}+E_{g}$

where $E_{f}$ is the metabolizable energy in the feed eaten each day, $R_{d}$ is the energy use efficiency which relates apparent energy use to available energy, $E_{m}$ is the energy needed for maintenance of the body, and $E_{g}$ is the energy needed for growth. By dividing Eq. (1) with $R_{d}$ and expanding the right side of the equation, we get:

$E_{f}=\frac{1}{R_{d}} \times\left(a \times B W^{b}+\left(\frac{\Delta \operatorname{Prot} \times 23.8^{\mathrm{MJ}} / \mathrm{kg}}{0.6}+\frac{\Delta \mathrm{Lip} \times 39.6^{\mathrm{MJ}} / \mathrm{kg}}{0.8}\right)\right)$

where $a$ is the linear metabolic constant corresponding to Maintenances, $B W$ is the body weight at the start of the day, $b$ is the metabolic maintenance exponent, $\Delta$ Prot is the growth of protein, and $\Delta$ Lip is the growth of lipid. $R_{d}$ was estimated for each feed with the "feed mix nutritional calculator" tool published by SEGES (2017) and the values of $a$ and $b$ were taken from van Milgen et al. (2008). It was chosen to model the $E_{m}$ based on the metabolic body mass over the protein mass since previous published and grey literature reported the body mass of the pig much more frequent than the protein. $\Delta$ Prot was estimated from nitrogen content in pigs slaughtered at different ages and transformed to crude protein by multiplying with 6.25 . $\Delta$ Lip was estimated by dividing $\Delta$ Prot with ProtLip, which was based on energy conservation in a simplified version of Wellock et al. (2004) updated to present pig performance. The $E_{m}$ was calculated for each day in the gilt and production pig stages with fast growth, accounting for the daily BW by assuming linear growth within a stage, and calculated for the average weight multiplied by the number of days for the sow stage with slower growth (see ESM, section 8.11 for energy implementation of traits). The LCA model was adapted on matrix form according to Heijungs and Suh (2002) (see Fig. 2):

$B \times A^{-1} \times f=g$

where $\mathbf{B}$ is the environmental matrix, containing impacts for each process, $\mathbf{A}$ is the technology matrix, containing the flow of resources inside the model, $\mathbf{f}$ is the functional unit, and $\mathbf{g}$ is the result matrix. The model was built in MATLAB R2017a (MathWorks 2017) with the B matrix imported from single inventories from SimaPro 8.5.2.0 (Pre Consultants 2017). A more thorough description of the matrix adaptation can be found in the ESM, section 8.9.

\subsection{Used data and compilation of correlation matrix}

Genetic variance and significant genetic correlations for pig traits were collected from 22 recently published articles on genetic variance and correlations in pigs (see ESM, section 8.12). The selected studies contained genetic correlations, additive genetic variance, and/or data which made it possible to calculate either of these under the assumption of additive genetic effects. Only studies conducted on common and commercially available modern fast-growing pig breeds raised indoor in Europe, North America, or Australia were included. Studies that did not list the number of pigs sampled were excluded. If a study investigated multiple breeds, each breed was included in the sample. If a study presented multiple results from different models, only results from the model the study recommended was included in the sample.

Trait correlations with measured or obvious high correlations were merged. Average daily feed intake was in this way replaced with ADG100, age at first farrowing was replaced with AgeMature, feed conversion ratio was replaced with residual feed intake (later transformed into Maintenance, see ESM, section 8.5), litter size and total litter weight for any parity number were replaced with LitterSize, and sow life time and sow lifetime piglets produced were replaced with AMPar. The correlation matrix was pooled with univariate weighting applying Fisher's $z$ transformation to reduce bias with different sample sizes and correlations (Furlow and Beretvas 2005). The variance was pooled from the studies weighted with sample size applying Bessel's correction (Upton and Cook 2014). If there was no known genetic correlation between any two traits, zero correlation was assumed. Since the pooled correlation matrix was not positive semidefinite, a small correction to the matrix was implemented to the nearest semidefinite matrix (Higham 2002). It was assumed that all GTs were normally distributed. 


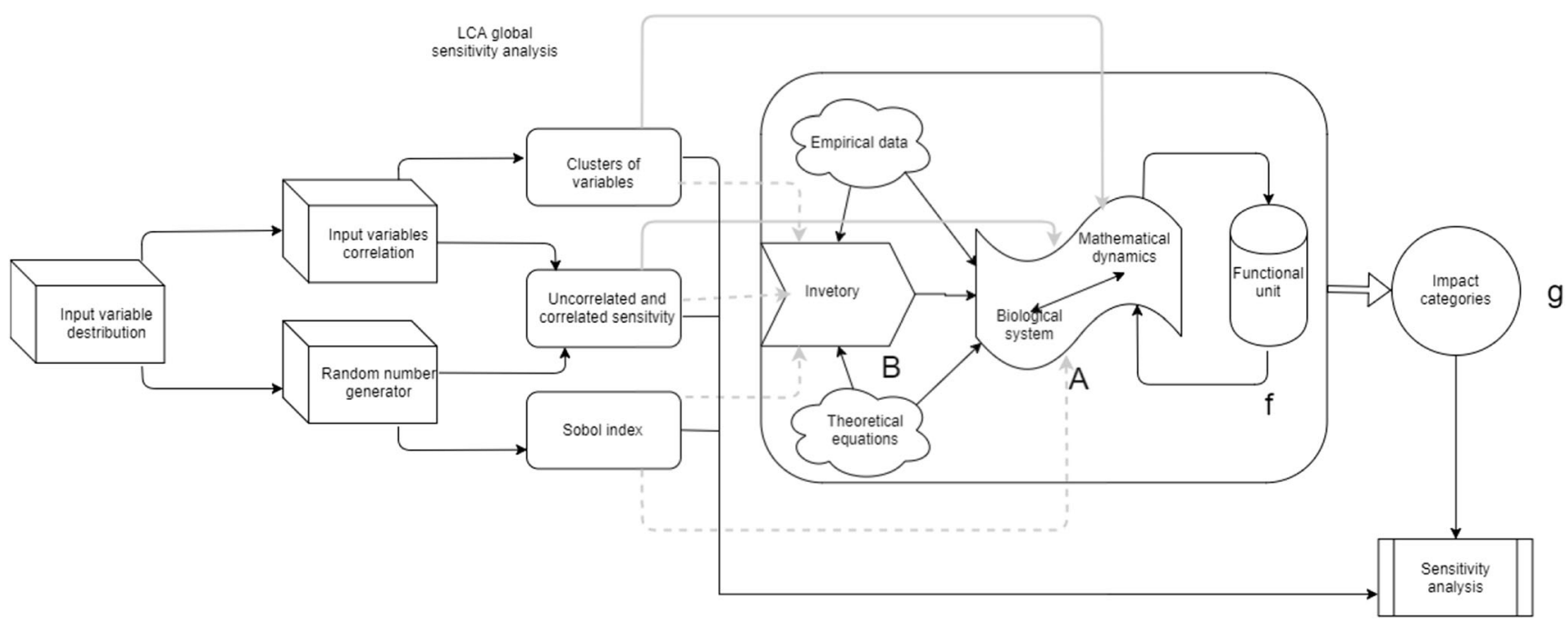

Fig. 2 Principal diagram of an LCA model on matrix form. The letters (B, $\mathbf{A}, \mathbf{f}, \mathbf{g})$ refer to the parts of the matrix LCA model of Eq. 3 in the text, where the inputs from the environmental matrix, $\mathbf{B}$, and the technology matrix, $\mathbf{A}$, are multiplied by the functional unit, $\mathbf{f}$, to produce the result, $\mathbf{g}$. Both empirical measurements and theoretical equations are used to fill out the $\mathbf{B}$ and A matrixes. Outside the bounds of the LCA model, the different

\subsection{Uncertainty and sensitivity}

To test the range of outputs from the model and to evaluate the effect of every included trait in the model, a number of uncertainty and sensitivity analyses were performed. This article follows the proposed definition by Saltelli et al. (2008), where the term uncertainty analysis (UA) comprises estimation of the variance of the model outcome and sensitivity analysis (SA) refers to estimation of relative contributions of individual variables to the overall variance.

\subsubsection{Sampling size for model outcomes uncertainty}

To take the genetic correlations between the traits into account when computing the average impact for the functional unit, and to estimate the uncertainty, the model was run multiple times with Latin hypercube sampling, and the mean and the variance were computed; this technique has been shown to converge with fewer samples than Monte-Carlo sampling (Groen et al. 2014). The optimal sample size was tested by the mean of the variance and the variance of the mean of 50 groups of samples. Increasing sample size was deemed unnecessary when the changes to the mean and variance became negligible with higher sample size (Ritter et al. 2011). The model was regarded as stable when the mean of the variance did not increase with increased sample size and the variance of the means was below $1 \%$ of the mean.

\subsubsection{Sensitivity analysis}

The sensitivity of different GTs for environmental impacts was tested using multiple methodologies for sensitivity elements in global sensitivity analysis and possible methods for sampling and scenarios are given. Solid grey lines illustrate methods used, dotted grey lines illustrate methods considered but not applied. The sampling process interacts with the outcome of the model to produce a result of the sensitivity analysis

analysis, as described below in brief. A more mathematical description can be found in the ESM, section 8.10. The methodologies accounted for genetic correlations between traits to a different extent.

\subsubsection{One-at-a-time sensitivity analysis}

A traditional way to estimate the sensitivity of the variables is to conduct a one-at-a-time sensitivity analysis (OAT) to test local sensitivity. The genetic variance found in the correlation matrix in section 2.8 was used to compute the standard deviation for each trait. Each trait (see Table 1) was changed by \pm 2 $\mathrm{SD}$ and the percent change from Baseline System value of each impact category was recorded.

\subsubsection{Cluster of traits sensitivity analysis}

Following the recommendations of Wei et al. (2015) to test the influence of interactions among GTs, a global sensitivity analysis was performed by first grouping the traits into clusters, followed by varying the clusters one at a time. This test is termed clusters of traits sensitivity analysis (COT) in the following text. To ensure that the traits which were most closely related to each other were grouped together, we constructed the clusters from the correlation matrix of the traits (see section 2.8). Here, the relation between the traits was based on the genetic correlation and the Euclidean distance was computed with the cosine relation, where a shorter distance reflects a higher similarity. Since negative genetic correlations between traits affect the outcome of the breeding strategy just as much as positive correlations, and the output of the cosine relation gets larger with larger negative numbers, the absolute 
correlation was used in this calculation. An upwards hierarchical clustering method was implemented in SAS 9.4 software (SAS Institute Inc. 2012) and the optimal number of clusters was chosen based on the pseudo $t$-squared index. For each cluster with more than one trait, all traits were varied by 0.2 intervals to $\pm 2 \mathrm{SD}$ according to the direction of internal correlations. Each cluster was varied independent of the other clusters.

\subsubsection{Total, uncorrelated, and correlated sensitivity index}

To estimate the influence of the genetic correlation between all the traits at once, a second global sensitivity analysis was performed by calculating the total, uncorrelated, and correlated sensitivity index (total SI, uncorrelated SI and correlated SI respectively) (Xu and Gertner 2008; Groen and Heijungs 2017), sampled with correlated Latin hypercube sampling. This technique estimates the fraction of the total model variance which can be associated with the variance in each individual trait (total SI), the fraction of the model variance which can be associated with the trait itself without interactions with other traits (uncorrelated SI), and the fraction of the model variance which can be associated with the interaction of the trait with other traits (correlated SI). This was done by first estimating the total SI and the uncorrelated SI, followed by estimation of the correlated SI by subtracting uncorrelated SI from total SI. The total SI will therefore be the sum of the correlated and uncorrelated SI; total and uncorrelated SI have values between zero and one, but correlated SI can range between plus one and minus one, since it accounts for the difference between the total and the uncorrelated sensitivity.

The sample size for the TSI, UCSI, and CSI was investigated with the mean of the variance and the variance of the mean of 20 groups of means and variances each based on 20 results from the investigated sample size. Results were calculated for sample sizes of $2^{5}$ to $2^{12}$, and the optimal sample size was predicted with regression for higher number of samples. The sample size was chosen when the changes to the mean of the variance were negligible with higher sample size (Ritter et al. 2011).

The results are presented in the order of increasing complexity: the model outcomes are presented first followed by the results of the local sensitivity analysis and thirdly, the outcome of the two global sensitivity analyses are presented.

\section{Results}

\subsection{Outcomes of the LCA model}

The outcomes of the LCA model are reported in Table 2 with 100 correlated samples produced with Latin hypercube sampling. The mean of variance of the model outcome reached a constant value, and the variance of the mean was $<0.01 \%$, <
$0.0001 \%,<10^{-6} \%,<0.01 \%$, and $<0.0001 \%$ for GWP, TAP, FEP, LU, and FRS, respectively, after 30 samples from correlated Latin hypercube sampling.

The production pig component contributed the majority of the environmental impacts, followed by the sow and the gilt system. TAP and FRS were more dominated by the sow component than the other impacts and LU had the highest contributions from the production pig system.

\subsection{One-at-a-time sensitivity analysis}

The results of the OAT are shown in Fig. 3. All environmental impacts were sensitive to maintenance $(12.8 \%, 18.8 \%, 11.5 \%$, $12.6 \%$ and $9.6 \% /-12.5 \%,-18.4 \%,-11.2 \%,-12.4 \%$, and $9.2 \%$ for GWP, TAP, FEP, LU, and FRS in + 2SD/- 2SD direction, respectively) with ADG100 being the second most influential trait for most EI in the - 2 SD change $(2.9 \%, 3.6 \%$, $3.2 \% 2.9 \%$, and $2.8 \%$ for GWP, TAP, FEP, LU, and FRS respectively in - 2 SD direction); BWMature, BWLossLactation, LitterSize, ProtLip, and AMPar had some influence (around $\pm 2 \%$ ), whereas ADG30, AgeMature, LactationFeed, PreWMort, and PostWMort had minimal influence on the model outcome $(< \pm 1 \%)$. ADG100, ADG30, BWlossLactation, ProtLip, and LitterSize reduced impacts when they were increased, whereas BWmaturity, Maintenance, and AMPar increased impacts when they were increased. TAP was the most sensitive environmental impact to trait variation; GWP, FEP, LU, and FRS had similar sensitivity to variation of traits among the EI for most traits.

\subsection{Correlated cluster sensitivity analysis}

The clustering produced five clusters with three clusters containing more than one trait. The multi-trait clusters can be seen in Table 3. Here, most traits had positive genetic correlations with other traits within the cluster, with the exception of ProtLip (cluster 1), LactationFeed (cluster 2), and AMPar (cluster 3) which had negative genetic correlations with the other traits inside their cluster. Cluster 1 contained traits associated with growth, cluster 2 contained traits associated with gestation and lactation, and cluster 3 contained traits associated with sow development and robustness.

The results of the COT can be seen in Fig. 4. The figure shows that when all traits in a cluster were changed by $\pm 2 \mathrm{SD}$, cluster 1 resulted in the highest change in environmental impacts, cluster 2 had an intermediate effect, and cluster 3 resulted in nearly no change in environmental impacts compared with the Baseline System. For clusters 1 and 2, it is clear that TAP was the most sensitive environmental impact to changes in all clusters. When all traits in cluster 1 were reduced by 2 SD according to their correlations (left side of Fig. 4), all environmental impacts were reduced. By reducing the cluster 1 traits in Fig. 4 by 2 SD according to their internal 
Table 2 The mean outcome of the model with pig genetic traits sampled 100 times with correlated Latin hypercube sampling and relative contribution to the environmental impacts for the three considered pig life stages: gilt (birth to first heat), sow (first heat up to 8th parity, including piglets until weaning), and production pig (pig for slaughter from weaning until slaughter weight). Impacts categories are global warming potential (GWP), terrestrial acidification potential (TAP), freshwater eutrophication potential (FEP), land use (LU), and fossil resource scarcity (FRS). Results are for $1-\mathrm{kg}$ live pig at farm gate. Standard deviations for the stochastic model are reported in parenthesises for absolute outcomes. Partial contributions are percent of Baseline Model result

\begin{tabular}{llllll}
\hline & \multicolumn{2}{l}{ Impact categories } & & & \\
\cline { 2 - 6 } & GWP & TAP & FEP & LU & FRS \\
\hline Model outcomes & $\begin{array}{c}4.18(0.28) \mathrm{kg} \\
\mathrm{CO}_{2} \mathrm{eq}\end{array}$ & $\begin{array}{c}40.9 \times 10^{-3}\left(4.00 \times 10^{-3}\right) \mathrm{kg} \\
\mathrm{SO}_{2} \mathrm{eq}\end{array}$ & $\begin{array}{c}552 \times 10^{-6}\left(33.4 \times 10^{-6}\right) \\
\mathrm{kg} \mathrm{P} \text { eq }\end{array}$ & $\begin{array}{c}4.12(0.27) \mathrm{m}^{2} \\
\text { land use }\end{array}$ & $\begin{array}{c}0.202(0.010) \mathrm{kg} \\
\text { oil eq }\end{array}$ \\
$\begin{array}{l}\text { Partial contribution of Baseline } \\
\text { System: }\end{array}$ & & & & $2.7 \%$ & $3.1 \%$ \\
Gilt & $2.4 \%$ & $2.0 \%$ & $2.6 \%$ & $11.1 \%$ & $26.1 \%$ \\
Sow & $18.7 \%$ & $22.9 \%$ & $17.8 \%$ & $86.2 \%$ & $70.8 \%$ \\
Production pig & $78.8 \%$ & $75.1 \%$ & $79.6 \%$ & & $2 \%$ \\
\hline
\end{tabular}

correlations, the TAP was reduced around $17 \%$ and other impacts were reduced around $10 \%$ relative to the Baseline System. Cluster 2 had reduced environmental impacts when all traits in the cluster were increased according to their internal correlations (right side of Fig. 4). By increasing cluster 2 traits in Fig. 4 by 2 SD according to their internal correlations, the TAP was reduced around $6 \%$ and other impacts were reduced around $4 \%$ compared with the Baseline System. Cluster 3 resulted in almost no change in EIs when the traits were changed, but had slightly reduced EIs when the traits in the cluster were increased according to their internal correlations (right side of Fig. 4). The EIs was slightly reduced by around $2 \%$ when the cluster 3 traits were increased by two SD.

\subsection{Sensitivity indices}

The total SI, uncorrelated SI, and correlated SI, produced with 20,000 samples, can be seen in Fig. 5. All EI categories showed similar SI for most traits, although TAP and LU showed slightly higher SI in dominant traits and FRS showed slightly lower SI than GWP and FEP for dominant traits. ProtLip $(0.39,0.42,0.40,0.44$, and 0.33 total SI for GWP, TAP, FEP, LU, and FRS, respectively) and Maintenance $(0.83,0.88,0.84,0.87$, and 0.74 total SI for GWP, TAP, FEP, LU, and FRS, respectively) were the traits with the highest total SI in all EIs, and were thereby responsible for most of the variance in the model outcome.
Fig. 3 The percent change to the environmental impacts compared with the Baseline System of global warming potential (GWP), terrestrial acidification potential (TAP), freshwater eutrophication potential (FEP), land use (LU), and fossil resource scarcity (FRS) when each genetic trait is varied by +2 (top) or -2 (bottom) SD one at a time. All trait abbreviations can be found in Table 1
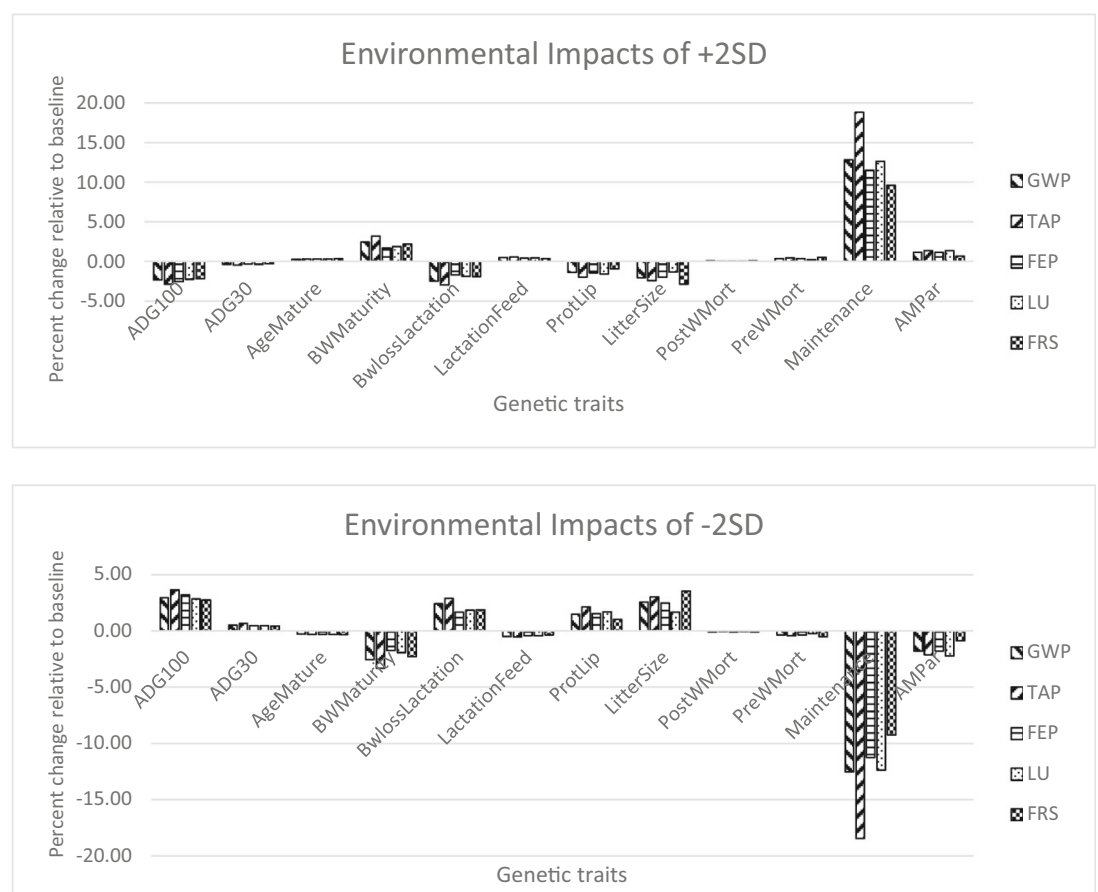
Table 3 Resulting clusters of traits from the upwards hierarchical clustering based on the genetic correlations; only clusters with more than one trait shown. Positive and negative sign indicates the direction of correlation and negative marked traits are changed opposite of traits marked positively within the same cluster

\begin{tabular}{lll}
\hline $\begin{array}{l}\text { Cluster 1: growth and } \\
\text { energy }\end{array}$ & $\begin{array}{l}\text { Cluster 2: } \\
\text { reproduction }\end{array}$ & $\begin{array}{l}\text { Cluster 3: sow } \\
\text { performance }\end{array}$ \\
\hline+ ADG100 & + BWLossLactation & + AgeMature \\
+ ADG30 & - LactationFeed & - AMPar \\
- ProtLip & + LitterSize & \\
+ Maintenance & + PreWMort & \\
\hline
\end{tabular}

BWMaturity, BWLossLactation, and LitterSize had a total SI of a few hundredths. The correlated SI contributed about half of the total SI for Maintenance and almost all of the total SI for ProtLip, so interactions for these traits with other traits were very important for explaining the variance in the model. BWLossLactation and LitterSize had nearly all their total SI from the correlated SI, and ADG100 had slightly negative correlated SI. Maintenance was the only trait with a major uncorrelated SI although ADG100 and BWMaturity also had minor contributions from the uncorrelated SI.

\section{Discussion}

The model presented in this paper illustrates the capabilities of a comprehensive method for predicting the influence of GTs on the environmental impacts of pig systems. This has been achieved by creating an LCA, which incorporates an animal energy model that can account for genetic changes to specific traits. By pooling genetic variance and correlation of traits from the global pig population, we demonstrated the potential influence of different traits on the environmental impacts. Further, by taking the genetic correlations between the traits into account to different degrees using a range of sensitivity analysis techniques, we illustrated the importance of considering genetic correlations among traits in pigs, when devising future strategies to reduce environmental impacts through breeding. The following sections discuss the outcome of the LCA model and sensitivity analysis, the methodological challenges faced by and arising from this work, and finally its implications for sensitivity analysis in LCA models.

\subsection{Outcomes of the LCA model}

The environmental impacts from the baseline model in this study are consistent with literature values (Halberg et al. 2010; Nguyen et al. 2011). The result of this model is presented as a mean and standard deviation of 100 correlated sample a) Growth and energy traits



b) Reproductive traits



c) Sow Performance



Fig. 4 Percentage change in environmental impacts when clusters of genetic traits were varied to $\pm 2 \mathrm{SD}$. a Growth and energy trait cluster composed of ADG100, ADG30, ProtLip, and Maintenance; b reproduction trait cluster composed of BWLossLactation, LactationFeed, LitterSize, and PreWMort; c sow performance trait cluster composed of AgeMature and AMPar. All trait abbreviations can be found in Table 1

runs and the SI was calculated based on 20,000 samples. As we have illustrated with the mean of variance and variance of mean, the correct sample size was essential to achieve accurate results without wasting excessive resources (Baldini et al. 2017; Mendoza Beltran et al. 2018). It was confirmed that the majority of all impacts comes from the production pig system, so pig LCA models only focusing on wean to slaughter is a reasonable approximation for the system boundary (Monteiro et al. 2016), but this assumption could underestimate some impact categories for the full production system with up to $29 \%$. 
Fig. 5 Total, uncorrelated, and correlated sensitivity index for genetic traits based on 20,000 samples for global warming potential, terrestrial acidification potential, freshwater eutrophication potential, land use, and fossil resource scarcity. The index on the $y$-axis is not directly comparable with the percent change on the previous figures, but tendencies can still be read and compared
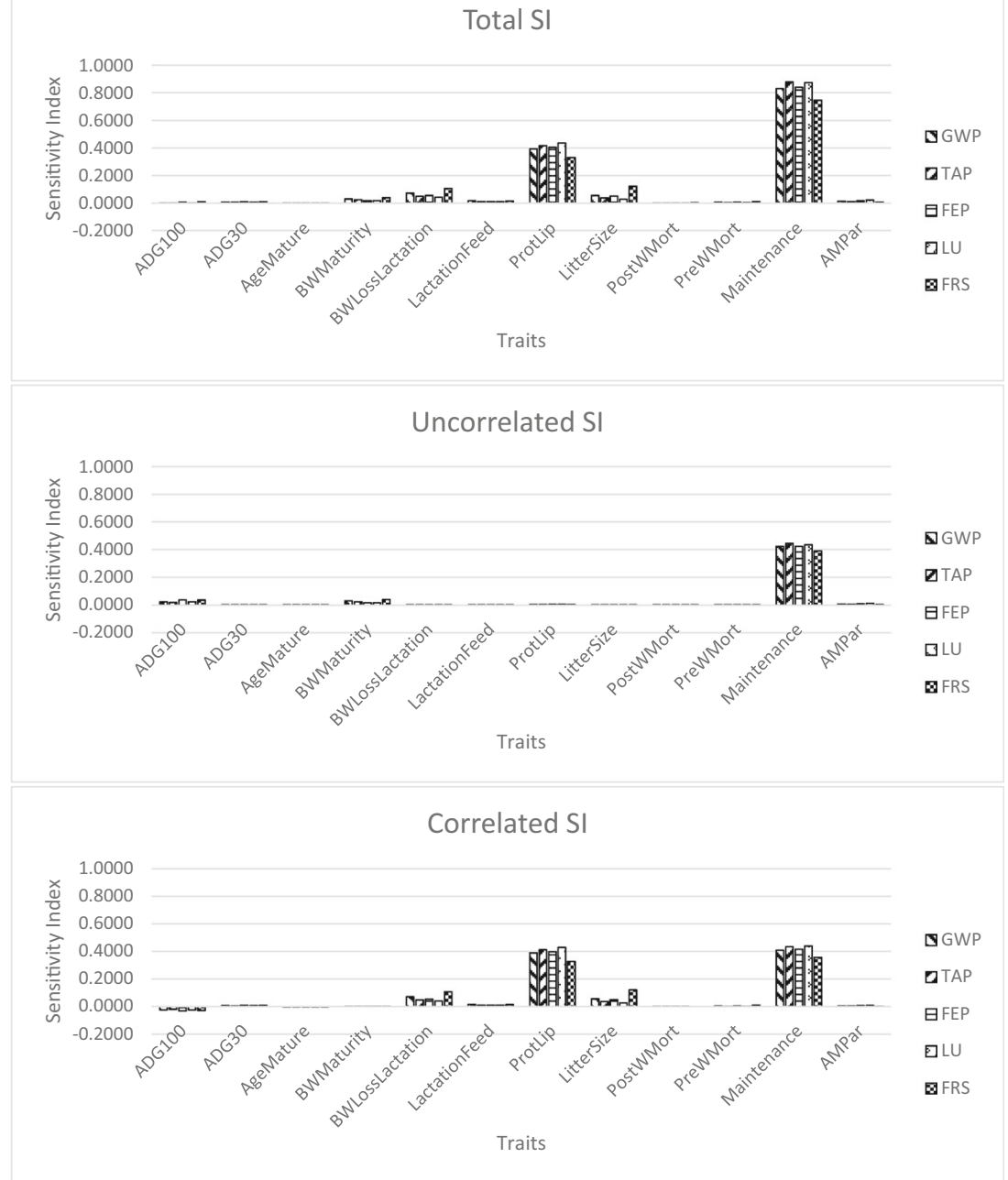

\subsection{Model sensitivity analysis}

The contrasting results of the different sensitivity analyses demonstrated the importance of the methodological approach when drawing conclusions regarding the potential of GTs to reduce environmental impacts. The results of the OAT sensitivity analysis showed the importance of Maintenance, ADG100, and some sow performance traits (see Fig. 3), whereas Maintenance and ProtLip were highlighted by the SI methodology (see Fig. 5) as good options to reduce environmental impacts. Both outcomes align with the focus of the breeding industry on growth rate and feed efficiency (PIC 2017; DANBRED 2018). However, when evaluating the potential of current breeding strategies to reduce the environmental impacts of pig production systems, it is critical to know which traits are improved when selecting for feed efficiency. This is important because some traits may have biological limits, for example lipid content in the body cannot go below a theoretical zero, and this needs to be recognized if such limits are being approached (Tallentire et al. 2018).
The OAT results did not suggest any environmental benefits from improving sow longevity and reducing pig mortality, since the OAT showed little sensitivity to changes in these traits, while AMPar actually increased environmental impacts when increased. The low sensitivity of environmental impacts to LitterSize and AMPar could be due to the high influence of management for outcomes in this phase of the production system, such as the choice of when to cull a sow (NagynéKiszlinger et al. 2013; Thekkoot et al. 2016). Further, only traits which affect major inputs and outputs of the LCA model, i.e. feed inputs, live weight pigs produced, and manure from pig systems (Mackenzie et al. 2015; McAuliffe et al. 2016), will have a major influence on the environmental impacts. Maintenance and ProtLip could therefore be expected to be influential since they affect feed intake for both the gilt, the sow, and the production pig. Traits like LitterSize and AMPar only directly influence the breeding phase of the production cycle for pigs when considered in an LCA model. Increases in AMPar increased environmental impacts because the higher $\mathrm{BW}$ as the sow increases in age requires higher feed intake without equivalent associated benefits in increased outputs, 
though this effect is small compared with other traits. Piglet mortality only has minor influence on the environmental impacts, since the invested feed to produce them is small compared with the full grown production pig. In a similar way, traits which only affects a short period in the life cycle (such as AgeMature and ADG30) or traits with only a small variance compared with the mean value (such as LactationFeed) can be expected to only have minor contribution to the outcome of the model.

Considering the genetic correlations in relation to the COT sensitivity analysis (Fig. 4), some classic breeding objectives may not be as effective as would be expected in reducing environmental impacts. For example, the COT suggested that, due to the negative correlations between on one hand ADG30 and ADG100 and on the other hand ProtLip, breeding for increased ProtLip is a much more effective strategy to reduce environmental impacts than increasing growth rate (see Table 3 and Fig. 4 cluster 1). Similarly, an increase in piglet mortality only has a minor effect on environmental impacts when compared with reductions in sow feed intake and higher litter size due to negative correlations between the traits in cluster 2. This could be due to some simplifications in the modelling process, where the interaction between sow and piglet performance was only included in the sampling process, and not as a causal effect implemented with deterministic equations. During the last decade, the pig production industry has moved towards higher sow replacement rates (AHDB Pork, SEGES, personal communication), though multiple publications have argued for lower sow replacement for economic and welfare reasons (Stalder et al. 2003; Onteru et al. 2011; Gruhot et al. 2017). In this study, sow longevity and its interaction with AgeMature had very little influence on the environmental impact categories tested for cluster 3 , which could be partly due to their negative correlation.

The total SI highlighted the sensitivity of the environmental impacts to changes in Maintenance and ProtLip; the sensitivity to other traits, including ADG100, was much lower. In a similar pig LCA study on correlated traits (Reckmann and Krieter 2015) also found that the environmental impacts of pig production systems were most sensitive to ProtLip but showed low sensitive to ADG100. ProtLip was one of the most, but ADG100 was one of the least, sensitive traits. This is in good agreement with the total SI where ProtLip was very sensitive and ADG100 was not very sensitive. The discrepancy between the outcome of the OAT and SI for ADG100, ProtLip, and AMPar illustrates the importance of accounting for genetic correlations between traits in pigs.

\subsection{Methodological challenges}

Due to necessity, many LCA studies implement a number of simplifications to reduce the amount of data needed to build a model. In livestock LCA models, a system is often described by static inputs and outputs, with feed intake based on feed conversion ratio and animal performance being independent of feed intake, e.g. Kebreab et al. (2016). In this study, we needed to investigate the effects of genetic changes in traits, and feed intake was therefore a function of performance and energy use. Further, we needed to integrate the sow system with the production pig system, as many of the traits frequently targeted by the pig breeding sector have major effects on both the production pig and the sow. To accurately model the effect of longevity traits of the sow, we needed to adapt the model to follow the sow from weaning to culling. This is different from the commonly applied methodology, where the reproductive system is modelled as an annual steadystate system and replacement rate accounts for the number of gilts imported into the system (Nguyen et al. 2011; Mackenzie et al. 2015).

A common argument for not including correlations in LCA modelling is the lack of data availability and the assumption that correlations have minor influence on the outcome (Heijungs 2010; Leinonen et al. 2016). We have shown in this paper that while published genetic correlations for pig traits may be limited in specific areas (i.e. we found no correlations between the trait BWMaturity and traits such as LitterSize, PostWMort and PreWMort), it was still possible through careful weighting to develop a correlation matrix. Instead of changing one variable at a time and accounting for correlations in each scenario (Reckmann and Krieter 2015), we utilized an unguided clustering technique, based on the pooled correlation matrix, to ensure the COT was not affected by personal bias. The limited availability of genetic variances and correlations in literature represented a significant challenge for this study. Conception rates, digestive efficiency, and constants related to the energy required for maintenance by the pigs were all input parameters to the model, but data on genetic correlations and variance of these traits were not available in literature. Major differences have, however, been suggested among pig population for digestive efficiency (Noblet et al. 2013) and heat production (Galassi et al. 2015; Kiarie et al. 2015) which most likely is in turn heavily reliant on maintenance. More studies with non-traditional pig trait genetic correlations, bridging sow longevity and performance, litter size and piglet performance, and grower/finisher performance, would be beneficial for future research on the effects of genetic changes to traits on environmental impacts.

Further, our work illustrated the very different requirements regarding sample size when computing the model uncertainty and the SI. Only 100 samples were required for sufficient precision in the uncertainty analysis, while the SI sensitivity analysis required 20,000 samples to achieve sufficient precision, necessitating multiple hours of computing and fostered a number of coding challenges related to reaching the limits of available computer power. For now, this still represents a barrier for the widespread adoption of global sensitivity analysis in 
complex LCA modelling as would be considered best practice for quantitative modelling (Saltelli et al. 2008).

\subsection{Sensitivity analyses in LCA}

The three sensitivity analyses implemented in this study illustrate different applications of the model. The OAT methodology highlighted the importance of every single trait independent of the other traits. Even though OAT is flawed with regard to correlations, the results of this analysis can show the size of the effect each single trait has on the environmental impact of the production system. This has the potential to be utilized to integrate environmental impact considerations and even objectives into breeding programs. The possibilities for using LCA modelling in this way have already been demonstrated for pig systems in relation to diet formulation (Mackenzie et al. 2016; Garcia-Launay et al. 2018).

The COT methodology reflects the genetic correlations among the traits better than OAT and still shows the possibilities of reducing EI in a more realistic way. However, COT is not capable of taking the size of the correlations into account, but can only differentiate between positive or negative correlations. The COT approach can effectively provide a sense check on any strategies devised for reducing environmental impacts, based on changing individual traits from the OAT method. Finally, the SI methodology highlights the capabilities of the traits to affect the environmental impacts, both when looking at individual traits, the interactions of one trait with another and the total influence the traits has on the model. One drawback of the SI method is that the output is an index, which is difficult to translate into an environmental impact reduction and does not show which direction a trait should be selected for to reduce impacts. However, the SI approach can clearly be used to identify the most important areas of focus when devising breeding strategies to reduce environmental impacts, without the need to fully integrate environmental objectives in the breeding model. Even though the SI analysis has been discussed before within modelling and engineering publications (e.g. Abbas and Morgenthal 2016; Kivekäs et al. 2018), and applied to a few animal systems (Godinot et al. 2014; Wolf et al. 2017), it has never been applied to animal LCA modelling. In this study, this method was an essential part of analysing the effect of the genetic correlations among traits.

Multiple global sensitivity analyses are available when conducting LCA models. The methods covered in this paper and possible supplementary sensitivity analyses are shown in Fig. 2. We optioned against using the Sobol index, and the expanded correlated version proposed by Jacques et al. (2006), since the first does not account for correlations and the second assumes there is no correlation between clusters, which in our case was not true. The SI sensitivity method was therefore chosen to account for correlations to their full extend. Since our study did not have access to correlations between the impacts in the primary production system of the feed ingredients in the B matrix, it was not possible to test the sensitivity of this part of the model.

Our study has illustrated the importance of an effective sensitivity analysis that fully encompasses the correlations among the stochastic variables. This is a central point for future studies, since most LCA studies still present limited data on the uncertainties of their models and few have performed sensitivity analysis (Hellweg and Canals 2014). This could partly be due to the limitations of typical modelling programs, which leaves little control of the modelling process and sensitivity test to the user (Mackenzie et al. 2016; Groen and Heijungs 2017; Tallentire et al. 2018). This study proposed a more flexible approach where exports from currently available LCA software were used as inputs to a model on matrix form in coding based modelling software.

The methodology illustrated in this paper is directly applicable to LCA models looking to determine the influence of traits and/or genetic changes on the environmental impacts of a given agricultural system. We have illustrated that a correlation matrix can be compiled from sparse data, and thereby weakened the argument of sparsity of available correlation data as a reason for ignoring correlations (Wei et al. 2015; Groen and Heijungs 2017). A more thoroughly and systematic testing of the sensitivity of biological models, as was done in this paper, could reduce the gap between simulations and reality if implemented into other systems. Further, the implementation of energy animal modelling, together with a similar protein estimation, could be used to predict realistic and optimal diets for more general animal systems than the typical diets often seen in LCA studies.

\section{Conclusions}

We have developed a novel methodology that is capable of identifying the implications of changing specific correlated traits for environmental impacts. This method is a robust way to evaluate the effects of genetic correlations in pigs, and we believe it could provide important insights if applied to other animal species. Our results showed that Maintenance, ADG100, and some sow performance traits were the most sensitive traits when tested one at a time, and ProtLip and Maintenance were the most sensitive traits when tested with global sensitivity. Further, we found that it was essential to prioritize between beneficial traits when breeding for a cluster of traits, and that grouped growth traits were most effective in reducing the environmental 
impacts from the system. The notable divergence in estimates according to different methodological implementations of sensitivity analysis indicates that accounting for genetic correlations are essential when modelling the potential of genetic changes to traits in pigs to reduce the environmental impacts of pig productions, and by extension other livestock. Of the common traits included in commercial breeding strategies, our study suggests a reduction in environmental impacts by improving growth and feed efficiency traits, while improvements in mortality and sow longevity only had minor effects on such impacts.

Funding information This research is part of the European SusPig and Feed a Gene projects. SusPig receives funding from the ERA-NET Sustainable Animals and the Department for Environment, Food and Rural Affairs of England. Feed a Gene receives funding from the European Union Horizon 2020 Programme for Research, Technological Development, and Demonstration under grant agreement no. 633531.

\section{Compliance with ethical standards}

Conflict of interest The authors declare that they have no conflict of interest.

Open Access This article is distributed under the terms of the Creative Commons Attribution 4.0 International License (http:// creativecommons.org/licenses/by/4.0/), which permits unrestricted use, distribution, and reproduction in any medium, provided you give appropriate credit to the original author(s) and the source, provide a link to the Creative Commons license, and indicate if changes were made.

\section{References}

Abbas T, Morgenthal G (2016) Framework for sensitivity and uncertainty quantification in the flutter assessment of bridges. Probabilistic Eng Mech 43:91-105

Baldini C, Gardoni D, Guarino M (2017) A critical review of the recent evolution of life cycle assessment applied to milk production. J Clean Prod 140:421-435

BSI (2011) PAS 2050 : 2011 specification for the assessment of the life cycle greenhouse gas emissions of goods and services. BSI Standards, UK. London

Capper JL (2011) The environmental impact of beef production in the United States: 1977 compared with 2007. J Anim Sci 89:4249-4261

Capper JL, Cady RA, Bauman DE (2009) The environmental impact of dairy production: 1944 compared with 2007. J Anim Sci 87:2160 2167

Cederberg C, Sonesson U, Henriksson M et al (2009) Greenhouse gas emissions from Swedish consumption of meat, milk and eggs 1990 and 2005. The Swedish Institute for Food and Biotechnology

DANBRED (2018) Breeding goal: focus on maximum genetic gain. https://danbred.com/en/avlssystem-uk/breeding-objectives-of-thefuture/. Accessed 4 Sep 2018

Dourmad JY, Etienne M, Valancogne A et al (2008) InraPorc: a model and decision support tool for the nutrition of sows. Anim Feed Sci Technol 143:372-386

Driver A (2017) Highlighting the differences - how UK welfare standards compare with our competitors. PIGWORLD
FAO (2016) Environmental performance of animal feeds supply chains: guidelines for assessment. Rome, Italy

FAO (2017) The future of food and agriculture -trends and challenges. Rome

FAO (2018) Environmental performance of pig supply chains: guidelines for assessment (version 1). Rome

FAOSTAT (2018) World, production animals/slaughtered meat, pig. http://www.fao.org/faostat/en/\#compare. Accessed 12 Sep 2018

Furlow CF, Beretvas SN (2005) Meta-analytic methods of pooling correlation matrices for structural equation modeling under different patterns of missing data. Psychol Methods 10:227-254

Galassi G, Malagutti L, Colombini S et al (2015) Nitrogen and energy partitioning in two genetic groups of pigs fed low-protein diets at $130 \mathrm{~kg}$ body weight. Ital J Anim Sci 14:293-298

Garcia-Launay F, Dusart L, Espagnol S et al (2018) Multiobjective formulation is an effective method to reduce environmental impacts of livestock feeds. Br J Nutr 120:1-12

Godinot O, Carof M, Vertès F, Leterme P (2014) SyNE: an improved indicator to assess nitrogen efficiency of farming systems. Agric Syst 127:41-52

Groen EA, Heijungs R (2017) Ignoring correlation in uncertainty and sensitivity analysis in life cycle assessment: what is the risk? Environ Impact Assess Rev 62:98-109

Groen EA, Heijungs R, Bokkers EAM, de Boer IJM (2014) Methods for uncertainty propagation in life cycle assessment. Environ Model Softw 62:316-325

Gruhot TR, Calderón Díaz JA, Baas TJ et al (2017) An economic analysis of sow retention in a United States breed-to-wean system. J Swine Heal Prod 25:238-246

Guinee J (ed) (2002) Handbook on life cycle assessment, 1st edn. Springer, Netherlands

Halberg N, Hermansen JE, Kristensen IS, Eriksen J, Tvedegaard N, Petersen BM (2010) Impact of organic pig production systems on $\mathrm{CO} 2$ emission, $\mathrm{C}$ sequestration and nitrate pollution. Agron Sustain Dev 30:721-731

Heijungs R (2010) Sensitivity coefficients for matrix-based LCA. Int J Life Cycle Assess 15:511-520

Heijungs R, Suh S (2002) The computational structure of life cycle assessment, 11th edn. Springer Science+Buisiness Media Dordrecht

Hellweg S, Canals LMI (2014) Emerging approaches, challenges and opportunities in life cycle assessment. Science 344:1109-1113

Herrero M, Henderson B, Havlík P, Thornton PK, Conant RT, Smith P, Wirsenius S, Hristov AN, Gerber P, Gill M, Butterbach-Bahl K, Valin H, Garnett T, Stehfest E (2016) Greenhouse gas mitigation potentials in the livestock sector. Nat Clim Chang 6:452-461

Higham NJ (2002) Computing the nearest correlation matrix - a problem from finance. Manchester

Huijbregts MAJ, Steinmann ZJN, Elshout PMF et al (2016) ReCiPe 2016: a harmonized life cycle impact assessment method at midpoint and enpoint level - Report 1 : characterization

IPCC (2006) Emissions from livestock and manure management. In: Guidelines for national greenhouse gas inventories. IPCC, p 89

Jacques J, Lavergne C, Devictor N (2006) Sensitivity analysis in presence of model uncertainty and correlated inputs. Reliab Eng Syst Saf 91: $1126-1134$

Kebreab E, Liedke A, Caro D, Deimling S, Binder M, Finkbeiner M (2016) Environmental impact of using specialty feed ingredients in swine and poultry production: a life cycle assessment. J Anim Sci 94:2664-2681

Kiarie E, Kim IH, Nyachoti CM (2015) Effect of genotype on heat production and net energy value of a corn-soybean meal-based diet fed to growing pigs. Vet Med (Praha) 60:489-498

Kivekäs K, Lajunen A, Vepsäläinen J, Tammi K (2018) City bus powertrain comparison: driving cycle variation and passenger load sensitivity analysis. Energies 11:1-26 
Knox RV (2016) Artificial insemination in pigs today. Theriogenology $85: 83-93$

Leinonen I, Williams AG, Kyriazakis I (2016) Potential environmental benefits of prospective genetic changes in broiler traits. Poult Sci 95: $228-236$

Mackenzie SG (2016) Modelling the environmental impacts of pig farming systems and the potential of nutritional solutions to mitigate them. Newcastle University

Mackenzie SG, Leinonen I, Ferguson N, Kyriazakis I (2015) Accounting for uncertainty in the quantification of the environmental impacts of Canadian pig farming systems. J Anim Sci 93:3130-3143

Mackenzie SG, Leinonen I, Ferguson N, Kyriazakis I (2016) Towards a methodology to formulate sustainable diets for livestock: accounting for environmental impact in diet formulation. Br J Nutr 115:18601874

Mackenzie SG, Leinonen I, Kyriazakis I (2017) The need for co-product allocation in the life cycle assessment of agricultural systems-is "biophysical" allocation progress? Int J Life Cycle Assess 22:128137

MacLeod M, Gerber P, Mottet A et al (2013) Greenhouse gas emissions from pig and chicken supply chains - a global life cycle assessment. Rome

MathWorks (2017) MATLAB 9.2.0.556344 (R2017a)

McAuliffe GA, Chapman DV, Sage CL (2016) A thematic review of life cycle assessment (LCA) applied to pig production. Environ Impact Assess Rev 56:12-22

Mendoza Beltran A, Prado V, Font Vivanco D, Henriksson PJG, Guinée JB, Heijungs R (2018) Quantified uncertainties in comparative life cycle assessment: what can be concluded? Environ Sci Technol 52: 2152-2161

Miller PS, Moreno R, Johnson RK (2011) Effects of restricting energy during the gilt developmental period on growth and reproduction of lines differing in lean growth rate: responses in feed intake, growth, and age at puberty. J Anim Sci 89:342-354

Monteiro ANTR, Garcia-Launay F, Brossard L, Wilfart A, Dourmad JY (2016) Effect of feeding strategy on environmental impacts of pig fattening in different contexts of production: evaluation through life cycle assessment. J Anim Sci 94:4832-4847

Nagyné-Kiszlinger H, Farkas J, Kövér G, Nagy I (2013) Selection for reproduction traits in Hungarian pig breeding in a two-way cross. Anim Sci Pap Reports 31:315-322

Nguyen TLT, Hermansen JE, Mogensen L (2011) Environmental assessment of Danish Pork. www.digisource.dk, ISBN: 978-87-91949-548

Noblet J, Gilbert H, Jaguelin-Peyraud Y, Lebrun T (2013) Evidence of genetic variability for digestive efficiency in the growing pig fed a fibrous diet. Animal 7:1259-1264

Oldenbroek K, van der Waaij L (2014) Textbook animal breeding animal breeding and genetics for BSc students. Wargeningen University and Research Centre, Wageningen

Onteru SK, Fan B, Nikkila MT et al (2011) Whole-genome association analyses for lifetime reproductive traits in the pig. J Anim Sci 89: 988-995

Pelletier N (2018) Changes in the life cycle environmental footprint of egg production in Canada from 1962 to 2012. J Clean Prod 176: $1144-1153$

Pelletier N, Ibarburu M, Xin H (2014) Comparison of the environmental footprint of the egg industry in the United States in 1960 and 2010. Poult Sci 93:241-255. https://doi.org/10.3382/ps.2013-03390

PIC (2017) The Camborough: efficiency, robustness, and prolificacy. The Industry-Leading economic Package

Poore J, Nemecek T (2018) Reducing food's environmental impacts through producers and consumers. Science 360:987-992

Pre Consultants (2017) SimaPro Release 8.5.2.0

Reckmann K, Krieter J (2015) Environmental impacts of the pork supply chain with regard to farm performance. J Agric Sci 153:411-421
Ritter FE, Schoelles MJ, Quigley KS, Klein LC (2011) Determining the number of simulation runs: treating simulations as theories by not sampling their behavior. In: Rothrock L, Narayanan S (eds) Humanin-the-loop simulations. Springer London, London, pp 97-116

Saltelli A, Ratto M, Andres T et al (2008) Global sensitivity analysis: the primer. John Wiley \& Sons Ltd, Chichester

SAS Institute Inc. (2012) SAS 9.4 TS Level aM2

SEGES (2012) Vådfoderkurver. http://130.227.75.183/Viden/Foder/ Opslagstavlen/Vaadfoderkurver.aspx\%0A.

SEGES (2013a) Fasefodring. https://svineproduktion.dk/Viden/I-stalden/ Foder/Foderstrategi/Fasefodring.

SEGES (2013b) FODRING AF DRÆGTIGE SØER. FODRING AF DRÆGTIGE SØER. Accessed 1 Oct 2018

SEGES (2017) Videnscententer for Svineproduktion Fodermiddeltabel

SEGES (2018) Svineproduktion: viden. https://svineproduktion.dk/ Viden.

SEGES Undsætingsstrategi,. http://svineproduktion.dk/Viden/I-stalden/ Management/Soeer/Udsaetningsstrategi.

Sloth NM (2000) 3-fasefodring af slagtesvin med differentieret fosfornorm

Sørensen G (2005) TØRFODER EFTER ÆDELYST TIL DIEGIVENDE SØER. In: Medd. nr. 686. https://svineproduktion. $\mathrm{dk} /$ Publikationer/Kilder/lu medd/2005/686.aspx. Accessed 1 Oct 2018

Springmann M, Clark M, Mason-D’Croz D, Wiebe K, Bodirsky BL, Lassaletta L, de Vries W, Vermeulen SJ, Herrero M, Carlson KM, Jonell M, Troell M, DeClerck F, Gordon LJ, Zurayk R, Scarborough P, Rayner M, Loken B, Fanzo J, Godfray HCJ, Tilman D, Rockström J, Willett W (2018) Options for keeping the food system within environmental limits. Nature 562:519-525

Stalder KJ, Stalder KJ, Lacy RC et al (2003) Financial impact of average parity of culled females in a breed-to-wean swine operation using replacement gilt net present value analysis. J Swine Heal Prod 11: 69-74

Szabó C, Jansman AJM, Babinszky L et al (2001) Effect of dietary protein source and lysine:DE ratio on growth performance, meat quality, and body composition of growing-finishing pigs. J Anim Sci 79: $2857-2865$

Tallentire CW (2018) Sustainability assessment of chicken meat production. Newcastle University

Tallentire CW, Leinonen I, Kyriazakis I (2016) Breeding for efficiency in the broiler chicken: a review. Agron Sustain Dev 36:1-16

Tallentire CW, Leinonen I, Kyriazakis I (2018) Artificial selection for improved energy efficiency is reaching its limits in broiler chickens. Sci Rep 8:1-10

Thekkoot DM, Kemp RA, Rothschild MF, Plastow GS, Dekkers JCM (2016) Estimation of genetic parameters for traits associated with reproduction, lactation, and efficiency in sows. J Anim Sci 94:45164529

Tribout T, Caritez JC, Gruand J, Bouffaud M, Guillouet P, Billon Y, Péry C, Laville E, Bidanel JP (2010) Estimation of genetic trends in French Large White pigs from 1977 to 1998 for growth and carcass traits using frozen semen. J Anim Sci 88:2856-2867

Upton GJG, Cook IT (2014) A dictionary of statistics. Oxford University Press, Oxford

van Milgen J, Valancogne A, Dubois S, Dourmad JY, Sève B, Noblet J (2008) InraPorc: a model and decision support tool for the nutrition of growing pigs. Anim Feed Sci Technol 143:387-405

Verge XPC, Dyer JA, Desjardins RL, Worth D (2009) Long-term trends in greenhouse gas emissions from the Canadian poultry industry. $\mathrm{J}$ Appl Poult Res 18:210-222

Wei W, Larrey-Lassalle P, Faure T, Dumoulin N, Roux P, Mathias JD (2015) How to conduct a proper sensitivity analysis in life cycle assessment: taking into account correlations within LCI data and interactions within the LCA calculation model. Environ Sci Technol 49:377-385 
Wellock IJ, Emmans GC, Kyriazakis I (2003) Modelling the effects of thermal environment and dietary composition on pig performance: model logic and concepts. Anim Sci 77:255-266

Wellock IJ, Emmans GC, Kyriazakis I (2004) Modeling the effects of stressors on the performance of populations of pigs 1. J Anim Sci 82: $2442-2450$

Wolf P, Groen EA, Berg W, Prochnow A, Bokkers EAM, Heijungs R, de Boer IJM (2017) Assessing greenhouse gas emissions of milk production: which parameters are essential? Int J Life Cycle Assess 22:441-455

Xu C, Gertner GZ (2008) Uncertainty and sensitivity analysis for models with correlated parameters. Reliab Eng Syst Saf 93:1563-1573

Publisher's note Springer Nature remains neutral with regard to jurisdictional claims in published maps and institutional affiliations. 